ANNALES

POLONICI MATHEMATICI

$81.2(2003)$

\title{
On the Noether exponent
}

\author{
by Anna Stasica (Kraków and Le Bourget du Lac)
}

Abstract. We obtain, in a simple way, an estimate for the Noether exponent of an ideal $I$ without embedded components (i.e. we estimate the smallest number $\mu$ such that $\left.(\operatorname{rad} I)^{\mu} \subset I\right)$.

1. Introduction. Let $\mathbf{k}[X]$ be the polynomial ring of $n$ variables over an algebraically closed field. The Nullstellensatz guarantees that for a given ideal $I \subset \mathbf{k}[X]$ there is a number $\mu$ such that $(\operatorname{rad} I)^{\mu} \subset I$. The smallest such $\mu$ is called the Noether exponent of the ideal $I$ and will be denoted by $\mu_{I}$. An estimate of this exponent was obtained by Kollár in $[\mathrm{K}]$. Subsequently several authors contributed to this problem, especially in the easier case when the ideal $I$ has only isolated components in its primary decomposition (see e.g. [CP], [FPT], [JOW], [STV], [AM]).

In this note we also consider the case without embedded components. We give a very simple method to obtain an estimate for the Noether exponent (Theorem 7) which is sharper than the results obtained in [FPT] and [JOW]. More precisely for the ideal $I=\left(f_{1}, \ldots, f_{k}\right)$, where $\operatorname{deg} f_{2} \geq \ldots \geq \operatorname{deg} f_{k} \geq$ $\operatorname{deg} f_{1}$ we show that

$$
\mu_{I} \leq \max _{i \in\left\{r_{1}, \ldots, r_{m}\right\}}\left\{\frac{\operatorname{deg} f_{1} \cdot \ldots \cdot \operatorname{deg} f_{i}}{d_{i}}\right\},
$$

where $r_{1}, \ldots, r_{m}$ are all possible codimensions of irreducible components of the zero set of the ideal $I$, and $d_{i}$ is the minimal degree of irreducible components of codimension $i$ of the variety given by the ideal $I$.

We conjecture that this estimate is also valid in the general case.

Acknowledgements. I am very grateful to Professor Zbigniew Jelonek for calling my attention to this problem and for many helpful discussions.

2000 Mathematics Subject Classification: 13C14, 14 R99.

Key words and phrases: Noether exponent, effective Nullstellensatz.

This paper is partially supported by the KBN grant number 2 PO3A 01722 . 
2. Preliminaries. We denote by $\mathbb{A}^{n}$ the affine space of dimension $n$ and by $\mathbf{k}[X]=\mathbf{k}\left[x_{1}, \ldots, x_{n}\right]$ the polynomial ring over the algebraically closed field $\mathbf{k}$. The zero set of an ideal $I$ is denoted by $V(I)$. For an algebraic set $Z \subset \mathbb{A}^{n}$ we consider the ideal $I(Z)=\left\{f \in \mathbf{k}[X] \mid f_{\mid Z}=0\right\}$.

Let $Z_{1}, \ldots, Z_{r} \subset \mathbb{A}^{n}$ be hypersurfaces and let $V$ be an irreducible component of $Z_{1} \cap \ldots \cap Z_{r}$. We say that $Z_{1}, \ldots, Z_{r}$ meet properly along $V$ or that this intersection is proper along $V$ if $\operatorname{dim} V=n-r$. Recall

Definition 1. Let $Z_{1}=\left\{F_{1}=0\right\}, \ldots, Z_{r}=\left\{F_{r}=0\right\} \subset \mathbb{A}^{n}$ be hypersurfaces meeting properly along the variety $V$. Then we define the index of intersection of $Z_{1}, \ldots, Z_{r}$ along $V$ to be the number

$$
i\left(Z_{1} \cdot \ldots \cdot Z_{r} ; V\right):=e_{\mathbf{k}[X]_{p}}\left(\left(F_{1}, \ldots, F_{r}\right)_{p}\right),
$$

where

$$
\frac{e_{\mathbf{k}[X]_{p}}\left(\left(F_{1}, \ldots, F_{r}\right)_{p}\right)}{n !} t^{n}+\ldots
$$

is the Hilbert-Samuel polynomial of $\mathbf{k}[X]_{p} /\left(F_{1}, \ldots, F_{r}\right)_{p}$ and $p=I(V)$ is the ideal of the variety $V$.

Remark 2. Since $\mathbf{k}[X]_{p}$ is a Cohen-Macaulay ring we have

$$
i\left(Z_{1} \cdot \ldots \cdot Z_{r} ; V\right)=\operatorname{length}\left(\mathbf{k}[X]_{p} /\left(F_{1}, \ldots, F_{r}\right)_{p}\right) .
$$

See e.g. [F, Example 7.1.10, p. 123].

We will need the following facts:

Theorem 3 (Associativity formula from [Na, (24.7)]). Let $(R, \mathfrak{m})$ be a local ring and let $I$ be an $\mathfrak{m}$-primary ideal generated by a system of parameters $f_{1}, \ldots, f_{n} \in \mathfrak{m}$. Let $\mathfrak{b}$ be the ideal generated by $f_{1}, \ldots, f_{r}$ for some $r \leq n$, and let $p_{i}$ be the minimal prime ideals of $\mathfrak{b}$ such that length $\left(p_{i}\right)=k$ and $\operatorname{dim} R / p_{i}=n-r$. Then

$$
e(I)=\sum_{i} e\left(\left(I+p_{i}\right) p_{i}\right) \cdot e\left(\mathfrak{b} R_{p_{i}}\right) .
$$

Theorem 4. Let $\Phi: \mathbf{k}^{n} \rightarrow \mathbf{k}^{n}$ be a generically finite polynomial mapping of geometric degree gdeg $\Phi$ (by geometric degree we mean the number of points in a generic fiber). Then for each $y \in \mathbf{k}^{n}$ the number of isolated points in the fiber $\Phi^{-1}(y)$ is not greater than $\operatorname{gdeg} \Phi$.

Proof. The statement is obvious for a quasi-finite mapping. The general case follows from the Stein factorization applied to the compactification of $\Phi$. Indeed, let $\Gamma=\operatorname{graph} \Phi$ and let $\bar{\Gamma} \subset \mathbb{P}^{n} \times \mathbf{k}^{n}$ be its closure. Consider the projection $\bar{f}: \bar{\Gamma} \rightarrow \mathbf{k}^{n}$. Due to the Stein factorization theorem there exist a normal variety $W$ and two morphisms, $q: \bar{\Gamma} \rightarrow W$ which has connected fibers and $u: W \rightarrow \mathbf{k}^{n}$ which is finite, such that $\bar{f}=u \circ q$. Moreover, gdeg $u=$ $\operatorname{gdeg} \Phi$. Consequently, every fiber $\bar{f}^{-1}(y)$ has no more than gdeg $u=\operatorname{gdeg} \Phi$ 
connected components. This implies that the number of isolated points in a fiber $\Phi^{-1}(y)$ is not greater than $\operatorname{gdeg} \Phi$.

We have a useful characterization of the index of proper intersection:

Proposition 5. Let $Z_{1}=\left\{F_{1}=0\right\}, \ldots, Z_{r}=\left\{F_{r}=0\right\} \subset \mathbb{A}^{n}$ be hypersurfaces given by polynomials $F_{i}$ which meet along $V$ properly. Let $H_{j}=V\left(\alpha_{j}\right)$ be the hyperplane given by a linear form $\alpha_{j}$ for $j \in\{1, \ldots, n-r\}$. Define $\Phi:=\left(F_{1}, \ldots, F_{r}, \alpha_{1}, \ldots, \alpha_{n-r}\right)$. If the intersection $Z_{1} \cap \ldots \cap Z_{r} \cap H_{1} \cap$ $\ldots \cap H_{n-r}$ is proper at a point $Q$, then

$$
i\left(Z_{1} \cdot \ldots \cdot Z_{r} ; V\right)=i\left(Z_{1} \cdot \ldots \cdot Z_{r} \cdot L ; Q\right)=\mu_{Q}(\Phi)
$$

for every linear subspace $L$ of dimension $n-r$ which meets $\bigcap_{i=1}^{r} Z_{i}$ transversely at $Q$. Here $\mu_{Q}(\Phi)$ denotes the multiplicity index of $\Phi$ at $Q$.

Proof. We follow [No]. Set $F=\left\{F_{1}, \ldots, F_{r}\right\}$ and $H=\left\{\alpha_{1}, \ldots, \alpha_{n-r}\right\}$. Clearly $F_{1}, \ldots, F_{r}$ form a system of parameters of the localization $\mathbf{k}[X]_{p}$, where $p$ is the ideal of the variety $V$. Since the hyperplanes $H_{j}$ for $j \in$ $\{1, \ldots, n-r\}$ meet $V$ transversely at $Q$, it follows that $\{F, H\}$ is a system of local parameters in the ring $\mathbf{k}[X]_{\mathfrak{m}_{Q}}$ and hence from the associativity formula we get

$$
e((F, H))=e(((F, H)+p) / p) \cdot e\left(\mathbf{k}[X]_{p} /(F)_{p}\right) .
$$

Since $(F, H)$ generate the maximal ideal in the local ring $\mathbf{k}[X]_{\mathfrak{m}_{Q}}$, we get $e(((F, H)+p) / p)=1$ and

$$
e(F, H)=e\left(\mathbf{k}[X]_{p} /(F)_{p}\right),
$$

which proves that $i\left(Z_{1} \cdot \ldots \cdot Z_{r} ; V\right)=i\left(Z_{1} \cdot \ldots \cdot Z_{r} \cdot L ; Q\right)$.

We will also need the following version of the Bézout Theorem.

Theorem 6 (Bézout Theorem, an affine version). Let $Z_{1}=\left\{F_{1}=0\right\}$, $\ldots, Z_{r}=\left\{F_{r}=0\right\} \subset \mathbb{A}^{n}$ be hypersurfaces given by polynomials $F_{i}$ which meet along $V_{1}, \ldots, V_{s}$ properly. Then

$$
\sum_{i=1}^{s} i\left(Z_{1} \cdot \ldots \cdot Z_{r} ; V_{i}\right) \operatorname{deg} V_{i} \leq \prod_{i=1}^{r} \operatorname{deg} F_{i} .
$$

Proof. According to the previous proposition the index of intersection is independent of the choice of a generic point $P$. Take generic hyperplanes $H_{j}$ given by linear forms $\alpha_{j}(j=1, \ldots, n-r)$ and set $d_{i}:=\operatorname{deg} V_{i}$ for $i=1, \ldots, s$.

Clearly the intersection $S:=\left(\bigcup_{i=1}^{s} V_{i}\right) \cap \bigcap_{j=1}^{n-r} H_{j}$ is a finite set. Let $\left\{P_{1}^{i}, \ldots, P_{d_{i}}^{i}\right\}:=V_{i} \cap S$ for $i \in\{1, \ldots, s\}$. Consider the map $\Phi:=\left(F_{1}, \ldots, F_{r}\right.$, $\left.\alpha_{1}, \ldots, \alpha_{n-r}\right)$. Note that it is generically finite and hence for a generic fiber $\Phi^{-1}(y)$ we have, by Theorem 4 and by the inequality of Rusek-Winiarski 
[є, p. 319]),

$$
\sum_{P \in \Phi^{-1}(y)} \mu_{P}(\Phi)=\operatorname{gdeg} \Phi \leq \prod_{i=1}^{r} \operatorname{deg} F_{i},
$$

where $\mu_{P}(\Phi)$ is the multiplicity of $\Phi$ at $P$. Since there are exactly $d_{i}$ points in $V_{i} \cap S$ we have

$$
\sum_{P \in \Phi^{-1}(y)} \mu_{P}(\Phi)=\sum_{i=1}^{s} \mu_{P_{1}^{i}}(\Phi) \cdot d_{i},
$$

and finally due to Proposition 5 we get

$$
\sum_{i=1}^{s} i\left(Z_{1} \cdot \ldots \cdot Z_{r} ; V_{i}\right) \operatorname{deg} V_{i} \leq \prod_{i=1}^{r} \operatorname{deg} F_{i} .
$$

3. Main result. Our main result is the following estimate.

TheOREM 7. Let $I=\left(f_{1}, \ldots, f_{k}\right)$ be an ideal generated by polynomials $f_{j} \in \mathbf{k}[X]$, where $\operatorname{deg} f_{2} \geq \ldots \geq \operatorname{deg} f_{k} \geq \operatorname{deg} f_{1}$. Assume that there is a primary decomposition $I=\bigcap_{i=1}^{m} q_{i}$ without embedded components, where $q_{i}$ are $p_{i}$-primary ideals. Set $r_{i}:=\operatorname{codim} V\left(q_{i}\right)$, and define $d_{t}:=\min \left\{\operatorname{deg} V\left(q_{i}\right) \mid\right.$ $\left.\operatorname{codim} V\left(q_{i}\right)=t\right\}$ for $t \in\left\{r_{1}, \ldots, r_{m}\right\}$. Then

$$
\mu_{I} \leq \max _{t \in\left\{r_{1}, \ldots, r_{m}\right\}}\left\{\frac{\operatorname{deg} f_{1} \cdot \ldots \cdot \operatorname{deg} f_{t}}{d_{t}}\right\} .
$$

To prove this theorem we will proceed by reduction to the case where the intersection along components of $V(I)$ is proper. The proof will be given in the next section.

Observe that for an ideal $I$ without embedded components we are able to find in this ideal a finite set $F$ of polynomials such that each component of $V(I)$ can be represented as a proper intersection of some hypersurfeces given by polynomials which lie in the set $F$. In fact we have the following

Lemma 8. Let $I=\left(f_{1}, \ldots, f_{k}\right)$ be an ideal with only isolated $p_{i}$-prime components $q_{i}$, say $\left(f_{1}, \ldots, f_{k}\right)=\bigcap_{i=1}^{m} q_{i}$, and define $r_{i}:=\operatorname{codim} V\left(q_{i}\right)$. Then there exists a family of polynomials

$$
\left\{\begin{array}{l}
F_{1}:=f_{1}, \\
F_{u}:=a_{u}^{u} f_{u}+\ldots+a_{k}^{u} f_{k} \quad \text { for } u \geq 2
\end{array}\right.
$$

where $a_{j}^{u} \in \mathbf{k}$, such that for each $i \in\{1, \ldots, m\}$ the intersection $V\left(F_{1}\right) \cap$ $\ldots \cap V\left(F_{r_{i}}\right)$ along $V\left(q_{i}\right)$ is proper.

Proof. We will construct such a family inductively. Obviously for $i$ such that $\operatorname{codim} V\left(q_{i}\right)=1$ the statement is true. Assume that for all $s<l \leq$ $\max \left\{r_{1}, \ldots, r_{m}\right\}$ we have polynomials $F_{s}$ such that the intersection $W_{s}:=$ $V\left(F_{1}\right) \cap \ldots \cap V\left(F_{s}\right)$ is proper along each component which does not lie 
in the set $V(I)$. Moreover, suppose that if there is $i \in\{1, \ldots, m\}$ such that $r_{i}=s$ then the hypersurfaces $V\left(F_{1}\right), \ldots, V\left(F_{s}\right)$ meet properly along $V\left(q_{i}\right)$. Consider a decomposition $W_{l-1}=W_{l-1}^{1} \cup \ldots \cup W_{l-1}^{s_{l-1}}$ into irreducible components. Take points $x_{l-1}^{p} \in W_{l-1}^{p} \backslash V(I)$ (for non-empty $W_{l-1}^{p} \backslash V(I)$ ). Since not all $f_{j}$ for $j=l, \ldots, k$ vanish at $x_{l-1}^{p}$, for generic $a_{j}^{l}$ the intersection $W_{l-1} \cap V\left(F_{l}\right)$ along all components of codimension $l$ is proper. Thus if there is $V\left(q_{j}\right)$ such that $r_{j}=l$ then clearly it must be contained in $W_{l-1} \cap V\left(F_{l}\right)$ and hence $V\left(F_{1}\right), \ldots, V\left(F_{l}\right)$ meet properly along this $V\left(q_{j}\right)$. Continuing this process, in a finite number of steps we obtain a family of polynomials $F_{u}$ such that for each $i$ the intersection $V\left(F_{1}\right) \cap \ldots \cap V\left(F_{r_{i}}\right)$ is proper along $V\left(q_{i}\right)$.

4. Estimates. In this section we give the proof of the main result, hence we work throughout under the assumptions and notation of Theorem 7 . First, consider the rings $\mathbf{k}[X]_{p_{i}} / I_{p_{i}}$ for $i \in\{1, \ldots, m\}$. Since the ideal $I$ has only isolated components we obtain for each $i$ an isomorphism

$$
\mathbf{k}[X]_{p_{i}} / I_{p_{i}} \cong \mathbf{k}[X]_{p_{i}}\left(q_{i}\right)_{p_{i}}=: R_{i} .
$$

Consider in the ring $R_{i}$ an increasing family of modules

$$
M_{s}^{i}(h):=\left(\left(q_{i}\right)_{p_{i}}: h^{s}\right)=\left\{g \in R_{i} \mid g h^{s} \in\left(q_{i}\right)_{p_{i}}\right\},
$$

for some $h \in \mathbf{k}[X]$ with $h_{\mid V(I)}=0$. We have

LEMMA 9. If $s$ is such that

$$
M_{0}^{i}(h) \subsetneq \ldots \subsetneq M_{s}^{i}(h)=M_{s+1}^{i}(h)=\ldots
$$

then $h^{s} \in q_{i}$.

Proof. Take the smallest $n \in \mathbb{N}$ such that $h^{n} \in\left(q_{i}\right)_{p_{i}}$ and assume that $n>s$. Clearly $h^{n-s-1} \in M_{s+1}^{i}(h)$ and hence also $h^{n-s-1} \in M_{s}^{i}(h)$, but this means that $h^{n-1} \in\left(q_{i}\right)_{p_{i}}$, contrary to the minimality of $n$. Thus $\frac{h^{s}}{1}=\frac{a}{b}$ for some $a \in q_{i}$ and $b \notin \operatorname{rad} q_{i}$. This means that $b h^{s} \in q_{i}$ and since the ideal $q_{i}$ is primary, $h^{s} \in q_{i}$.

Define

$$
s_{i}(h):=\min \left\{s \mid M_{s}^{i}(h)=M_{s+1}^{i}(h)\right\}, \quad s_{i}:=\max \left\{s_{i}(h) \mid h_{\mid V(I)}=0\right\} .
$$

This is well defined since $R_{i}$ is the Artin ring. Finally define $s$ as the maximum of $s_{i}$ for $i \in\{1, \ldots, m\}$. Then we have the following inequalities:

LEMMA 10. $\mu_{I} \leq s \leq \operatorname{length}\left(R_{i}\right)$ for $i$ such that $s_{i}=s$. Consequently, $\mu_{I} \leq \max _{i \in\{1, \ldots, m\}}\left\{\right.$ length $\left.\left(R_{i}\right)\right\}$.

Proof. Take $h$ such that $h_{\mid V(I)}=0$. Then $h=0$ on each $V_{i}$ and hence $h^{s} \in q_{i}$ for each $i \in\{1, \ldots, m\}$ by Lemma 9 . This proves the first inequality. The second one is a consequence of the definition of the length. 
Proof of Theorem 7. We choose polynomials $F_{u}$ as in Lemma 8. Denote by $Z_{u}$ the hypersurface $V\left(F_{u}\right)$. Since for each $i=1, \ldots, m$ the intersection $Z_{1} \cap \ldots \cap Z_{r_{i}}$ is proper along $V\left(q_{i}\right)$, Remark 2 yields

$$
\text { length }\left(\mathbf{k}[X]_{p_{i}} /\left(F_{1}, \ldots, F_{r_{i}}\right)_{p_{i}}\right)=i\left(Z_{1} \cdot \ldots \cdot Z_{r_{i}} ; V_{i}\right) .
$$

Using the affine version of the Bézout Theorem we get

$$
i\left(Z_{1} \cdot \ldots \cdot Z_{r_{i}} ; V_{i}\right) \leq \frac{\operatorname{deg} F_{1} \cdot \ldots \cdot \operatorname{deg} F_{r_{i}}}{d_{r_{i}}} \leq \frac{\operatorname{deg} f_{1} \cdot \ldots \cdot \operatorname{deg} f_{r_{i}}}{d_{r_{i}}} .
$$

Clearly

$$
\operatorname{length}\left(R_{i}\right) \leq \operatorname{length}\left(\mathbf{k}[X]_{p_{i}} /\left(F_{1}, \ldots, F_{r_{i}}\right)_{p_{i}}\right),
$$

hence finally due to Lemma 10 we obtain

$$
\mu_{I} \leq \max _{i \in\left\{r_{1}, \ldots, r_{m}\right\}}\left\{\frac{\operatorname{deg} f_{1} \cdot \ldots \cdot \operatorname{deg} f_{i}}{d_{i}}\right\}
$$

Note that for a set-theoretic complete intersection we have at once (see $[\mathrm{PT}]$ )

Corollary 11. If an ideal $I=\left(f_{1}, \ldots, f_{r}\right)$ is a set-theoretic complete intersection, then

$$
\mu_{I} \leq \frac{\operatorname{deg} f_{1} \cdot \ldots \cdot \operatorname{deg} f_{r}}{\min _{i \in\{1, \ldots, s\}}\left\{\operatorname{deg} X_{i}\right\}}
$$

where $V(I)=\bigcup_{i=1}^{s} X_{i}$.

The next corollary is a generalization of a result from $[\mathrm{CP}]$.

Corollary 12. Let $f=\left(f_{1}, \ldots, f_{n}\right)$ be a polynomial mapping such that $f^{-1}(0)$ is a finite, non-empty set. Set $\mu:=\max \left\{\mu_{a}(f) \mid a \in f^{-1}(0)\right\}$, where $\mu_{a}(f)$ is the local multiplicity of the map $f$ at the point a. Then for each polynomial $g \in \mathbb{C}[X]$ such that $g_{\mid f^{-1}(0)}=0$, we have $g^{\mu} \in\left(f_{1}, \ldots, f_{n}\right)$.

Finally let us state the following

Conjecture. Let $I=\left(f_{1}, \ldots, f_{k}\right)$ be an ideal generated by polynomials $f_{j} \in \mathbf{k}[X]$, where $\operatorname{deg} f_{2} \geq \ldots \geq \operatorname{deg} f_{k} \geq \operatorname{deg} f_{1}$. Let $\bigcap_{i=1}^{m} q_{i}=I$ be a primary decomposition. Set $r_{i}:=\operatorname{codim} V\left(q_{i}\right)$ and define $d_{t}:=\min \left\{\operatorname{deg} V\left(q_{j}\right) \mid\right.$ $\left.\operatorname{codim} V\left(q_{j}\right)=t\right\}$ for $t \in\left\{r_{1}, \ldots, r_{m}\right\}$. Then

$$
\mu_{I} \leq \max _{t \in\left\{r_{1}, \ldots, r_{m}\right\}}\left\{\frac{\operatorname{deg} f_{1} \cdot \ldots \cdot \operatorname{deg} f_{t}}{d_{t}}\right\} .
$$

\section{References}

[AM] R. Achilles and M. Manaresi, A footnote to a result of Sturmfels-Trung-Vogel on the effective Nullstellensatz, An. Ştiinţ. Univ. Ovidius Constanţa Ser. Mat. 5 (1997), no. 2, 1-8. 
[CP] P. Cassou-Noguès et A. Płoski, Un théorème des zéros effectif, Bull. Polish Acad. Sci. Math. 44 (1996), 61-70.

[FPT] A. Fabiano, A. Płoski and P. Tworzewski, Effective Nullstellensatz for strictly regular sequences, Univ. Iagell. Acta Math. 38 (2000), 164-167.

[F] W. Fulton, Intersection Theory, Springer, 1998.

[JOW] W. Jarnicki, L. O'Carroll and T. Winiarski, Ideal as an intersection of zerodimensional ideals and the Noether exponent, ibid. 39 (2001), 139-146.

[K] J. Kollár, Sharp effective Nullstellensatz, J. Amer. Math. Soc. 1 (1988), 963-975.

[€] S. Łojasiewicz, Introduction to Complex Analytic Geometry, PWN, Warszawa, 1988 (in Polish).

[Na] M. Nagata, Local Rings, Interscience Publ., New York, 1962.

[No] K. Nowak, Improper intersections in complex analytic geometry, Dissertationes Math. 391 (2001).

[PT] A. Płoski and P. Tworzewski, Effective Nullstellensatz on analytic and algebraic varieties, Bull. Polish Acad. Sci. Math. 46 (1998), 31-38.

[STV] B. Sturmfels, N. V. Trung and W. Vogel, Bounds on degrees of projective schemes, Math. Ann. 302 (1995), 417-432.

Institute of Mathematics

Jagiellonian University

Reymonta 4

30-059 Kraków, Poland

E-mail: anna.stasica@im.uj.edu.pl
Laboratoire de Mathématiques Université de Savoie Campus scientifique F-73376 Le Bourget du Lac Cedex, France

Reçu par la Rédaction le 20.2.2002

Révisé le 12.7.2002 et 12.11.2002 